\title{
Consultative meeting to discuss ways to promote the use of seasonal influenza vaccine among high-risk groups in the Eastern Mediterranean Region ${ }^{1}$
}

Citation: Consultative meeting to discuss ways to promote the use of seasonal influenza vaccine among high-risk groups in the Eastern Mediterranean Region. East Mediterr Health J. 2021;27(1):96-97. https://doi.org/10.26719/2021.27.1.96

Copyright (C) World Health Organization (WHO) 2021. Open Access. Some rights reserved. This work is available under the CC BY-NC-SA 3.0 IGO license (https://creativecommons.org/licenses/by-nc-sa/3.o/igo).

\section{Introduction}

Influenza is a major cause of morbidity and mortality worldwide. Each year, seasonal influenza causes up to 650000 deaths and millions of people are hospitalized (1). Moreover, influenza pandemics can have a major impact on health and society as a whole. A sustainable seasonal influenza programme or policy not only reduces the disease burden but strengthens health systems and leads to better pandemic preparedness (1). Since it is conducted annually, seasonal influenza vaccination allows countries to develop, practice and refine vaccine approv$\mathrm{al}$, distribution and delivery plans, each year. Increasing national, regional and global efforts to expand the use of seasonal vaccines provides economic and public health benefits today, while developing systems for pandemic response in the future.

The importance of influenza vaccination has been highlighted in World Health Assembly resolution WHA56.19 on prevention and control of influenza pandemics and annual epidemics (2003) (2), the Global Action Plan for Influenza Vaccines (2006-2016) (3), the Pandemic Influenza Preparedness Framework (2011) (4) and World Health Assembly resolution WHA70(10) on Review of the Pandemic Influenza Preparedness Framework (2017) (5).

The capacity of countries in the WHO Eastern Mediterranean Region in surveillance and pandemic planning has greatly increased over the past decade. However, the Region continues to have some of the lowest rates of vaccine utilization globally. The countries of the Region are highly diverse with respect to their influenza vaccination programmes: some have high-quality robust annual programmes, some have small programmes they wish to expand, while others have yet to introduce the vaccine.

To address these issues a one-day consultative meeting was held in Casablanca, Morocco on 19 November 2019 (6) to discuss ways to promote the use of seasonal influenza vaccine among high-risk groups in the Eastern Mediterranean Region. The meeting focused on identifying challenges and opportunities for the introduction and expansion of influenza vaccination policies and programmes in the Region.

The objectives of the meeting were to:

- review the current landscape of influenza vaccination in the Region;

- review ongoing initiatives related to influenza vaccination and preparedness that are relevant to seasonal vaccination programme development;

- discuss key challenges to influenza vaccination programme growth in the Region;

- discuss tools and approaches to accelerating the creation of an evidence base for the development of national vaccine policies; and

- identify opportunities for regional collaboration to expand influenza prevention in the Region.

\section{Summary of discussions}

Despite the expansion of vaccine use globally, vaccines are still underused in many low- and middle-income countries, and in many countries that have vaccination policies, vaccine programmes remain weak. Political and technical challenges to vaccine expansion persist. These include negative perceptions of the relative value, affordability and availability of vaccines, regulatory obstacles, a need for national policies and a lack of operational plans to implement programmes.

In addition to these challenges, vaccine expansion can be technically difficult due to lack of information on disease burden, lack of an infant-focus among target groups and the need to deliver the programme annually, as well as difficulties in measuring impact, communicating value, matching supply and demand and integrating seasonal influenza vaccination into routine immunization programmes.

\section{Recommendations}

To WHO

- Creating a mechanism for regional cooperation towards greater influenza prevention and control through increased use of influenza vaccine;

- supporting countries in the Region with adequate

\footnotetext{
This summary is extracted from the Summary report on the One day consultative meeting to discuss ways to promote the use of seasonal influenza vaccine among high-risk groups in the Eastern Mediterranean Region, Casablanca, Morocco, 16 November 2019 (https://applications.emro.who.int/ docs/WHOEMCSR286E-eng.pdf?ua=1).
} 
epidemiological and laboratory surveillance data to conduct seasonal influenza burden estimation studies in support of better public health decision-making on seasonal influenza vaccine introduction; and

- ensuring the availability of appropriate influenza vaccination campaign materials translated into local languages in all countries of the Region.

\section{To Member States}

- Increasing the national evidence base by conducting influenza disease burden estimation studies and developing appropriate policy briefs;

- improving access to vaccination through introduction of policies on vaccination implementation by nurses, mobile teams and at pharmacies;

- increasing communication messages about influenza vaccines through campaigns, social media or other platforms, ensuring that communications are targeted at high-risk groups;

- reducing the price of influenza vaccination through national subsidies or by participating in regional pooled procurement; and

- advocating for a more effective partnership between the public and private sectors to promote seasonal influenza vaccine uptake and improve coverage rates.

\section{References}

1. World Health Organization. Influenza (seasonal). Geneva: World Health Organization; 2018 (https://www.who.int/news-room/ fact-sheets/detail/influenza-(seasonal).

2. World Health Organization. Prevention and control of influenza pandemics and annual epidemics. Geneva: World Health Organization; 2003 (https://apps.who.int/iris/handle/10665/78320).

3. World Health Organization. Global Action Plan for Influenza Vaccines (2006-2016). Geneva: World Health Organization; 2019 (https://apps.who.int/iris/bitstream/handle/10665/329300/9789241515634-eng.pdf?sequence=1\&isAllowed=y).

4. World Health Organization. Pandemic Influenza Preparedness Framework (2011). Geneva: World Health Organization; 2011 (https://www.who.int/influenza/resources/pip_framework/en/).

5. World Health Organization. Pandemic Influenza Preparedness Framework for the sharing of influenza viruses and access to vaccines and other benefits: progress in implementing decision WHA70(10) (2017) on Review of the Pandemic Influenza Preparedness Framework: report by the Director-General. Geneva: World Health Organization; 2018 (https://apps.who.int/iris/ handle/10665/276465).

6. World Health Organization Regional Office for the Eastern Mediterranean (WHO/EMRO). One day consultative meeting to discuss ways to promote the use of seasonal influenza vaccine among high-risk groups in the Eastern Mediterranean Region, Casablanca, Morocco, 16 November 2019. Cairo: WHO/EMRO; 2020 (https://applications.emro.who.int/docs/WHOEMCSR286E-eng. pdf?ua=1). 